\title{
High-throughput fabrication of right-angle prism mirrors with selective metalization by two-step 3D printing and computer vision alignment
}

\author{
Andrea Bertoncini ${ }^{\mathrm{a}}$, Gheorghe Cojoc $^{\mathrm{c}}$, Jochen Guck ${ }^{\mathrm{b}, \mathrm{c}}$, and Carlo Liberale ${ }^{\mathrm{a}}$ \\ ${ }^{a}$ King Abdullah University of Science and Technology \\ ${ }^{b}$ Max-Planck-Institut für Lichtwissenschaft \\ ${ }^{\mathrm{c}} \mathrm{TU}$ Dresden
}

\begin{abstract}
We propose a dual step $\mu$-3D printing (two-photon lithography) strategy, which is the $3 \mathrm{D}$ version of the classic lithography etching-mask strategy, to achieve selective gold metallization for the realization of reflective micro-optics. We apply this strategy to obtain right-angle prism micro-mirrors used to create dynamic counterpropagating optical traps for biological samples in a holographic optical trapping setup with a single low-NA objective. We print the prisms on standard glass coverslips to create integrated optical trapping chips. We show the automatization of alignment between prisms and masks by a computer vision software.
\end{abstract}

Keywords: 3D printing, micro-optics, Two-photon lithography, optical tweezers

\section{INTRODUCTION}

$\mu$-3D printing based on two-photon lithography is a powerful technique to create three-dimensional polymeric microstructures with sub-micron resolution. ${ }^{1}$ In particular, this method can be used to realize high-quality 3D printed micro-optics, whose size can be as small as few micrometers - a capability that is unmatched by other optics manufacturing techniques. ${ }^{2,3}$ Typically, 3D printed micro-optics can be based on light refraction, ${ }^{2}$ reflection $^{4}$ or diffraction. ${ }^{5}$ The combination of 3D printed micro-optical elements based on different optical mechanism can easily realize sophisticated optical devices. ${ }^{6-8}$ Reflective micro-optics offer the advantage of achromaticity, compactness, and the possibility to create broadband polarization phase retarders. ${ }^{9}$ When a reflective micro-optic element is designed to work in air as a medium, it is generally possible to exploit the total internal reflection (TIR) mechanism, because the condition for TIR is satisfied over a wide range of incidence angles, typically between $90^{\circ} 41^{\circ}$. However, when the reflective micro-optic element has to work in a liquid medium, e.g. in water immersion, the TIR condition is satisfied for a narrower range of incidence angles, e.g. between $90^{\circ}$ and $61^{\circ}$ for water immersion, and the TIR mechanism may no longer be exploited. Therefore, a localized metal coating on the designed reflective surface is required to realize certain water immersion microoptics, such as a right angle prism mirror, that is supposed to work at around $45^{\circ}$ incidence angle.

Here, we propose a dual step $\mu$-3D printing strategy to achieve selective metalization of micro-optic elements. Our approach can be considered as a 3D version of the standard 2D lithography etching mask strategy. In particular, here we show the selective metalization of the slanted face of right-angle micro-prisms to create micromirrors. Remarkably, we automated an alignment procedure - needed for the third step of our method (Fig. 2) - with a computer vision algorithm, to speed up the procedure and increase the throughput of the fabrication process. Right angle micro-mirrors might be used in future to create dynamic counterpropagating optical traps for biological samples in a holographic optical trapping setup with a single low-NA objective. ${ }^{10}$ Counterpropagating optical traps are an important optical trapping configuration for the study of living cells. ${ }^{5,11,12}$ The concept of such a device is rendered and explained in Fig. 1; two opposing micro-mirrors are printed on standard glass coverslips, to create devices that can be used with any holographic optical tweezers setup and are compatible with advanced microscopies.

Further author information: (Send correspondence to A.B)

A.B.: E-mail: andrea.bertoncini@kaust.edu.sa 


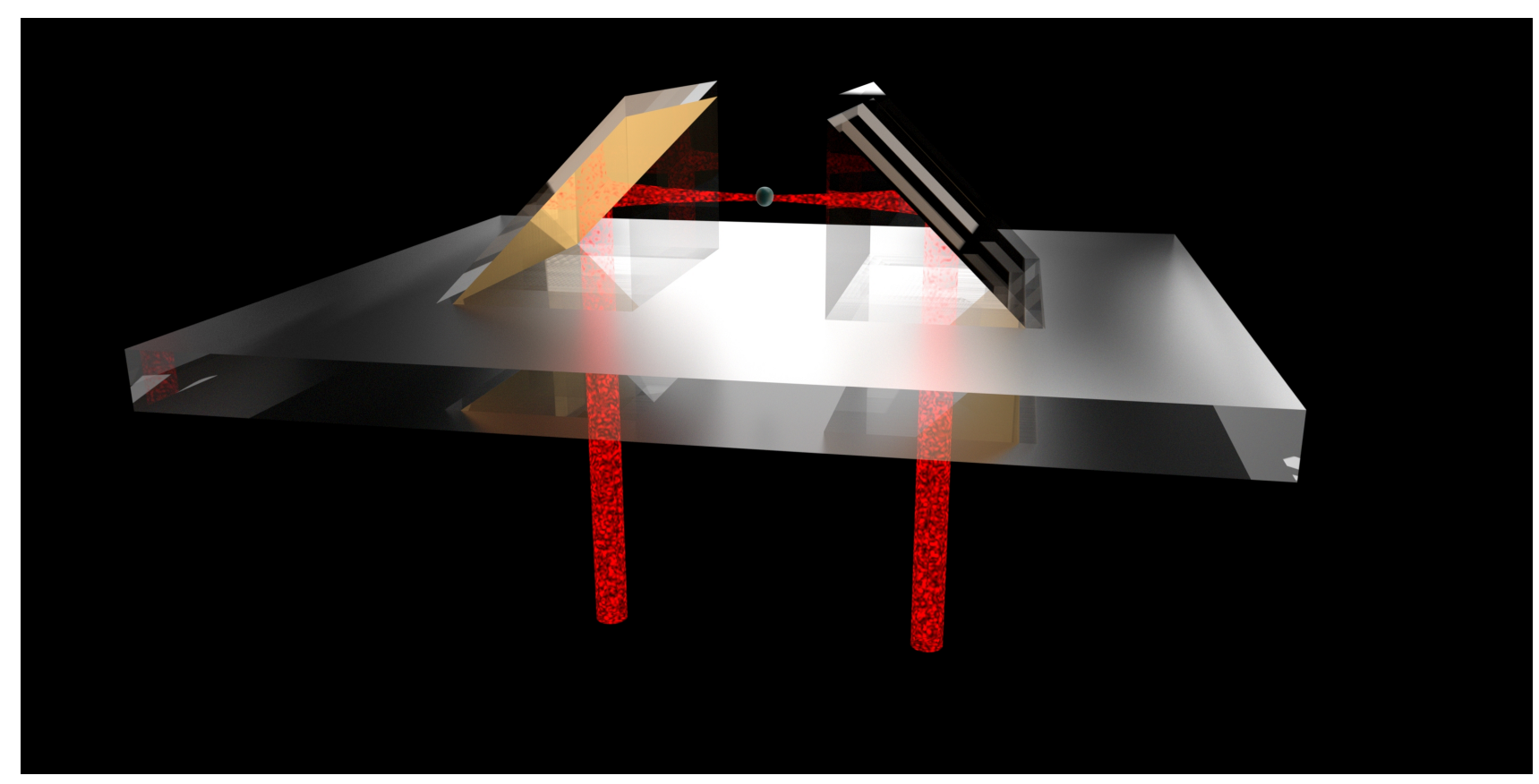

Figure 1. Counterpropagating beam optical tweezers with 3D printed right angle micro-mirrors Rendered illustration of two right angle micro-mirrors that redirect two parallel beams to create a counterpropagating beam laser trap. A dielectric bead is optically trapped in the volume between the micro-mirrors.

\section{METHODS}

We 3D printed the right angle micro-prisms using a commercially available Two-Photon Lithography system (Photonic Professional GT, Nanoscribe GmbH). The used photoresist was the proprietary IP-S (Nanoscribe $\mathrm{GmbH}$ ), which provides smooth surfaces and fast printing of relatively large volumes. The fabrication procedure of the right angle micro-mirrors with selective metal deposition consisted of the following four steps; The first step of the fabrication process was the 3D printing of right angle micro-prisms on a $170 \mu \mathrm{m}$ thick glass coverslip. The micro-prisms were 3D printed with a fine slicing of $0.1 \mu \mathrm{m}$, to have a optically smooth faces. We developed the sample by immersing it for 10 minutes in the mrDev600 developer and for 10 minutes in Isopropanol. In the second step of the process, we deposited an uniform $30 \mathrm{~nm}$ thick layer of gold on the coverslip — which includes the 3D printed micro-prisms - by sputtering with physical vapor deposition (step 2 in Fig. 2). At this stage, the coverslip would be unusable for optical trapping or transmission illumination microscopy, because all the faces of the micro-prisms are covered with gold. In the third step of the process, to maintain the deposited gold layer only on the slanted face of the micro-prisms, we 3D printed a $5 \mu \mathrm{m}$ thick protective layer of the IP-S photoresist on top of the prisms (step 3 in Fig. 2), followed by a development procedure as done for the step 1. Finally,a gold etching step removes the deposited gold from the whole coverslip, except the previously protected area, leaving a final device with a high-quality gold mirror on the tilted face of the micro-prisms (step 4 in Fig. 2).

In the step 3 of the fabrication process, an accurate 3D alignment between the micro-prisms - 3D printed in step 1 - and the protective polymer layer is crucial for a successful protection of the gold layer on the slanted surface of the micro-prisms.

This step is cumbersome and time consuming if done manually, and its automation is highly desirable towards an high-throughput production of such devices. Thus, to automate the alignment process, we developed a computer vision algorithm that automatically aligns the polymer protective layer on top of the corresponding micro-prism, without the supervision of an operator. The Nanoscribe 3D printer is essentially based on an inverted optical microscope, whose camera can be used to image the substrate after the gold coating (step 2 in 


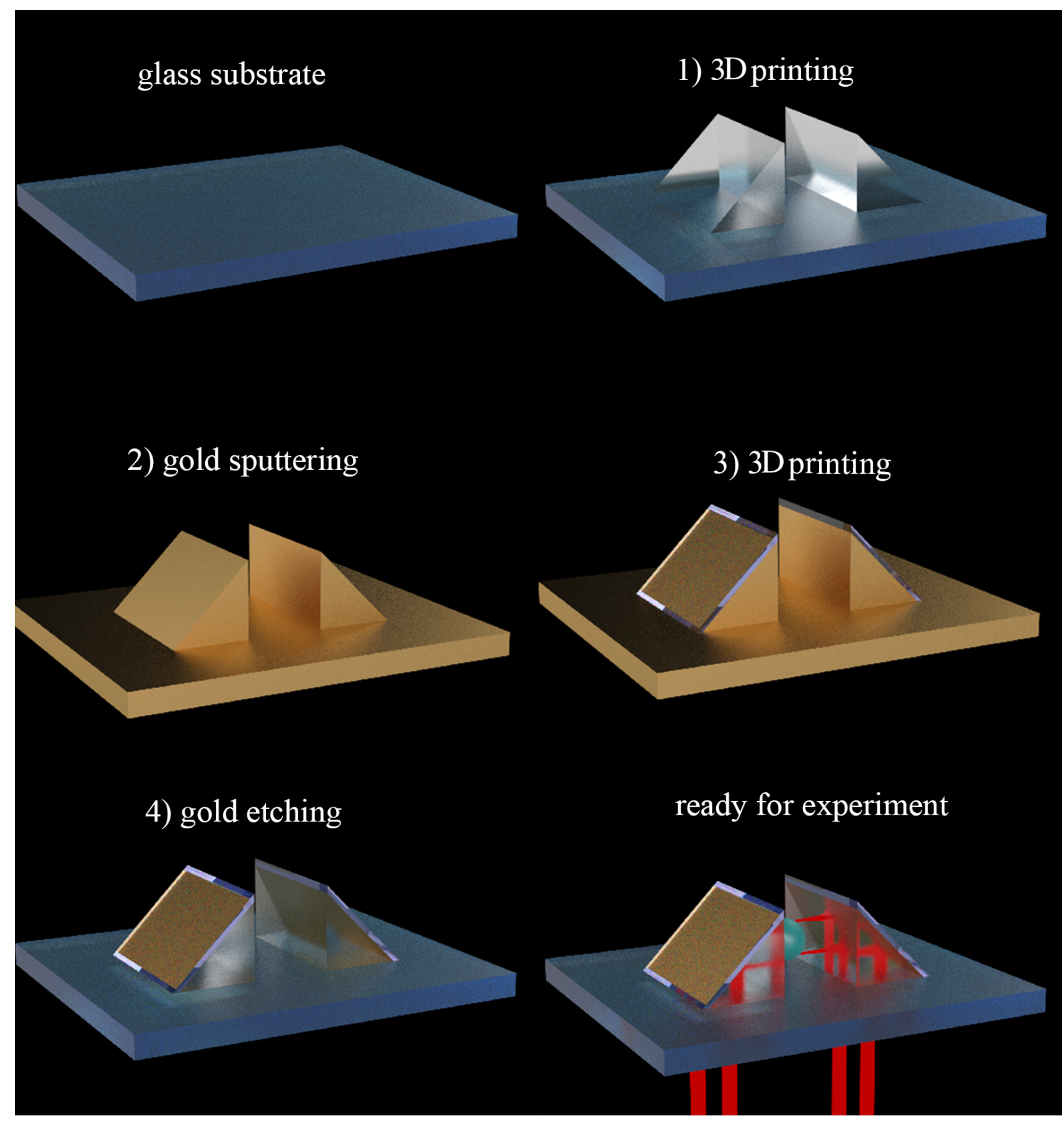

Figure 2. Fabrication procedure to create right-angle prism mirror with selective metalization. 
Fig. 2), search for the micro-prisms, identify the edges of the micro-prisms and automatically align and 3D print the protective layer. We achieved this through a Python script which interacts with the Nanoscribe ServerMode. By using this software and by paralleling all the steps of development, sputtering and etching, we can produce 10 devices with 5 pairs of micro-mirrors on each device, in the total time of 4 hours.

The steps of the algorithm are the following:

(a) The stage of the optical microscope starts scanning the sample in a spiral path until the camera finds the micro-prisms, then aligns them in the center of the field of view.

(b) The collected image is converted to a binary image.

(c) The binary image is processed to make it more sharp and defects free, through an erosion and dilation algorithm.

(d) A corner detection algorithm finds the coordinates of the corners of the base of each micro-prism, through a Harris corner detection algorithm; next, it sends the coordinates to the 3D printer software to create the $3 \mathrm{D}$ pattern for the 3D fabrication of the protective polymer layer on top of the slanted face of the micro-prisms.

The steps of this algorithm are illustrated in Fig. 3

\section{RESULTS}

We successfully fabricated right angle micro-mirrors with good optical quality. Fig. 4 shows the Scanning Electron Microscopy (SEM) image of one couple of micro-prisms at the step 2 (Fig. 2) of the fabrication process. The slanted face of the micro-prism that will be covered in gold at the end of the fabrication process (Fig. 2) is digitally colored in yellow for illustration purposes in Fig. 4.

We 3D printed a pair of micro-mirrors with a micro-post added in the middle (Fig. 5c) to demonstrate that the right angle micro-mirrors work in water immersion condition. We obtained a neat image of the micro-post from a side view, while imaging through the micro-mirrors with a 10x $0.2 \mathrm{NA}$ microscope objective and in reflection illumination configuration (Fig. 5a).

\section{DISCUSSION AND CONCLUSION}

We devised a method for the selective metalization of 3D printed micro-optics, by the selective inclusion of a metal layer though a two step process. This method is automated with a computer vision algorithm.

Other methods that could be exploited to achieve three dimensional metallic reflective micro-optics could be the electroplating in a $\mu-3 \mathrm{D}$ printed template ${ }^{13}$ or the direct $\mu-3 \mathrm{D}$ printing of metal by photoinduced electron transfer. ${ }^{14}$ The good optical quality of the side view through the micro-mirrors demonstrates that this device is suitable to be used to create dynamic counterpropagating optical traps in a holographic optical trapping setup. 


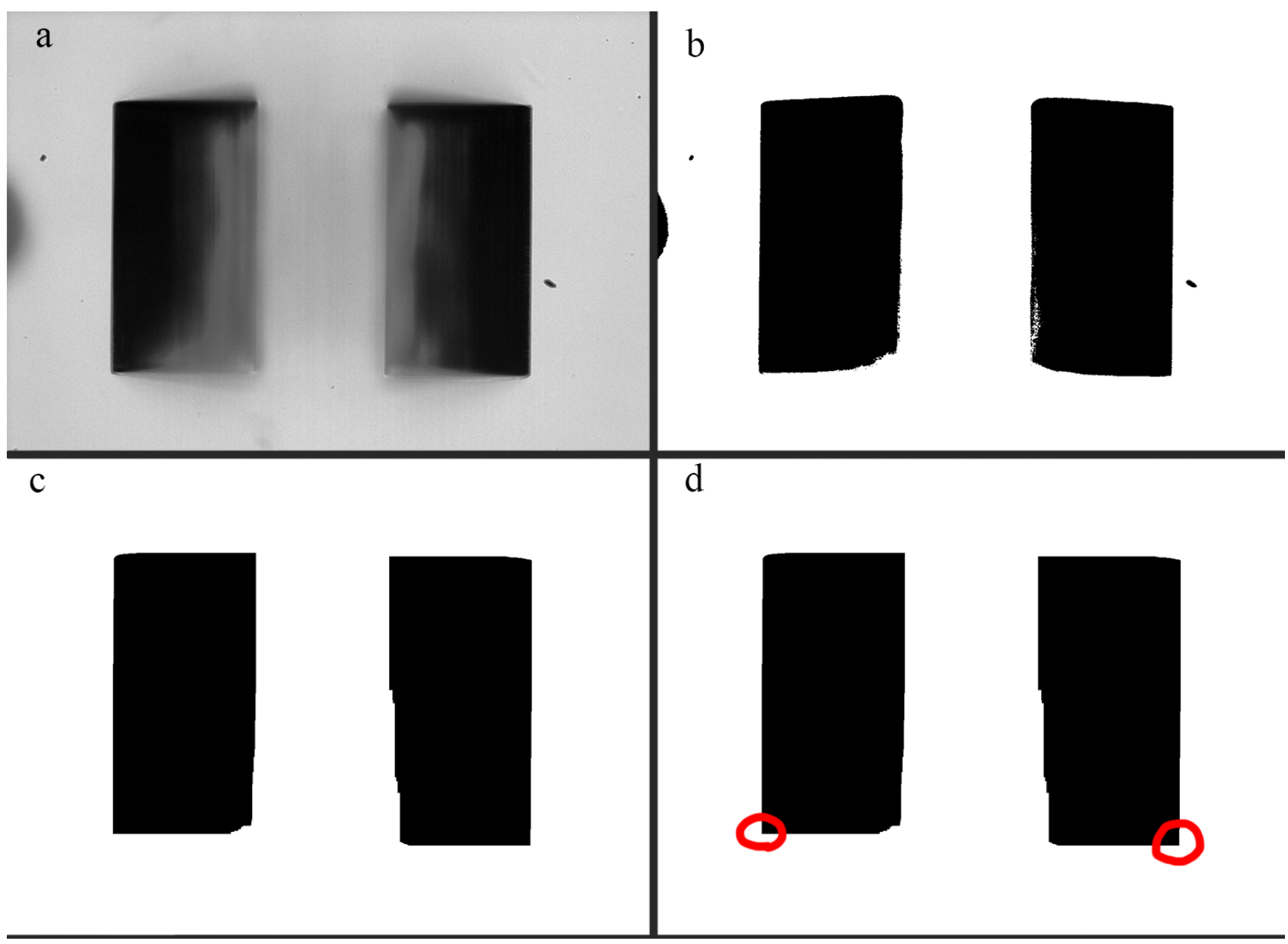

Figure 3. Steps of the computer vision algorithm for automated alignment of the 3D printed protective layer. 


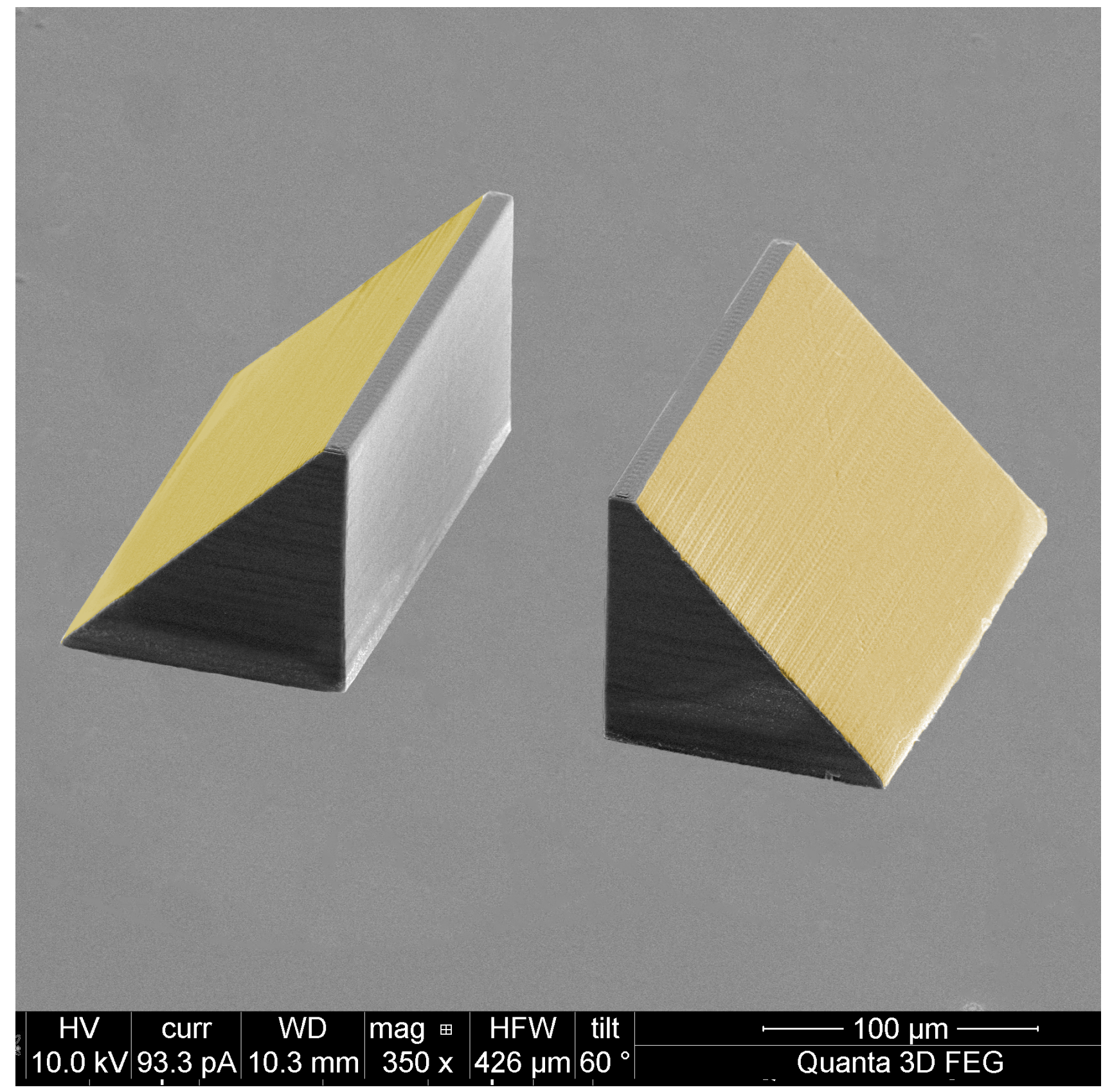

Figure 4. Digitally coloured Scanning Electron Microscopy of the prisms at the step 2 of the fabrication. 


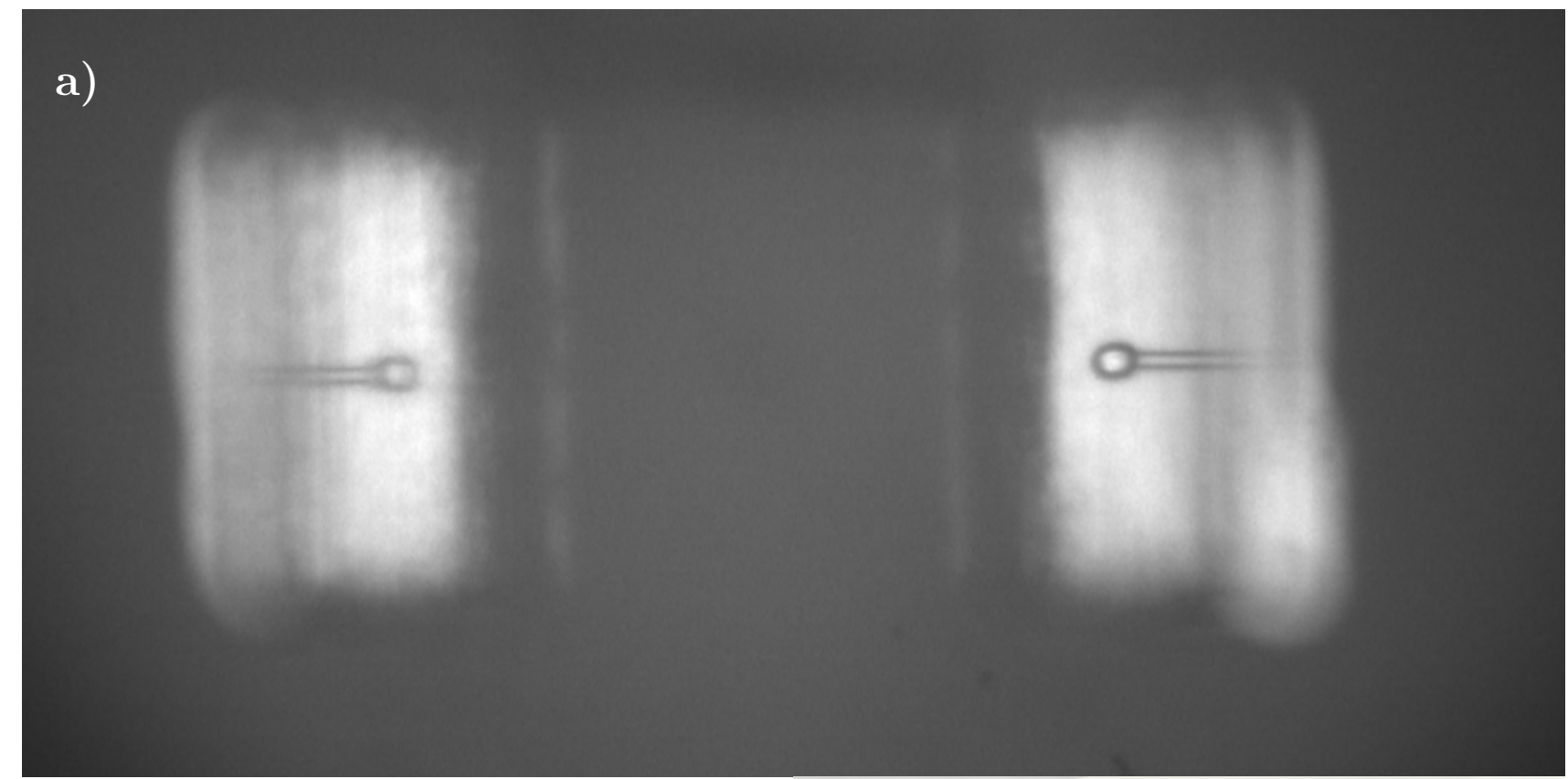

b)

c)
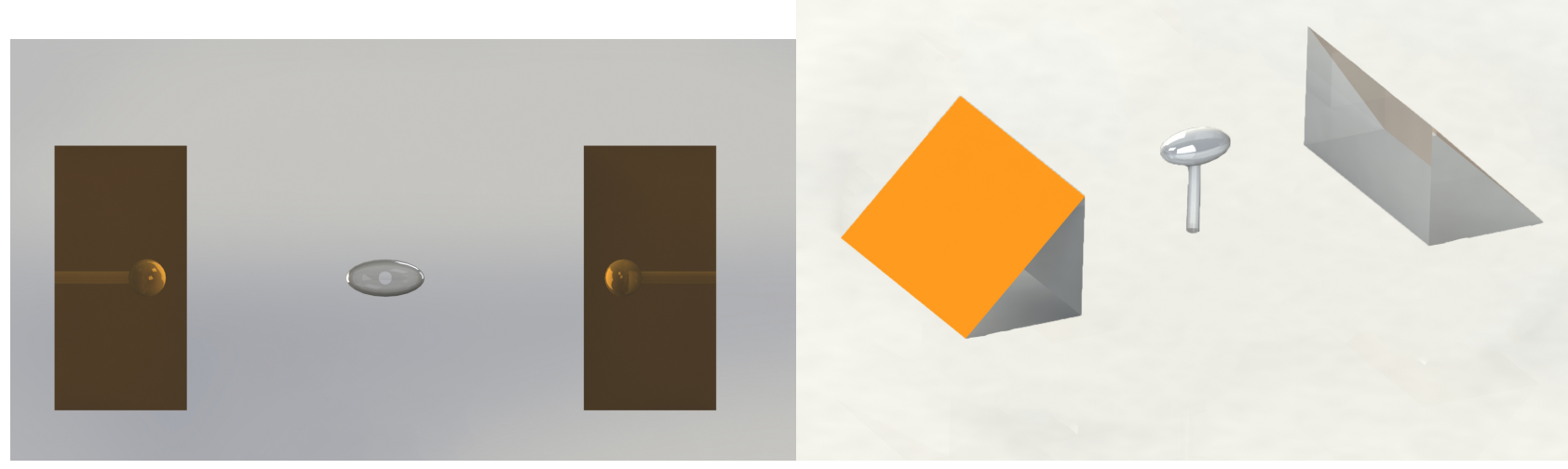

Figure 5. Side imaging proof of principle of a 3D printed pedestal+bead in the middle of prisms a) Optical image view from the bottom b) Rendered bottom view c) Prism + bead assembly render viewed from the top 


\section{REFERENCES}

[1] Hohmann, J. K., Renner, M., Waller, E. H., and von Freymann, G., "Three-dimensional $\mu$-printing: An enabling technology," Advanced Optical Materials 3(11), 1488-1507 (2015).

[2] Gissibl, T., Thiele, S., Herkommer, A., and Giessen, H., "Two-photon direct laser writing of ultracompact multi-lens objectives," Nature Photonics 10(8), 554 (2016).

[3] Varapnickas, S., Žukauskas, A., Brasselet, E., Juodkazis, S., and Malinauskas, M., "3d microoptics via ultrafast laser writing: Miniaturization, integration, and multifunctionalities," in [Three-Dimensional Microfabrication Using Two-Photon Polymerization], 445-474, Elsevier (2020).

[4] Liberale, C., Cojoc, G., Bragheri, F., Minzioni, P., Perozziello, G., La Rocca, R., Ferrara, L., Rajamanickam, V., Di Fabrizio, E., and Cristiani, I., "Integrated microfluidic device for single-cell trapping and spectroscopy," Scientific reports 3, 1258 (2013).

[5] Asadollahbaik, A., Thiele, S., Weber, K., Kumar, A., Drozella, J., Sterl, F., Herkommer, A. M., Giessen, H., and Fick, J., "Highly efficient dual-fiber optical trapping with 3d printed diffractive fresnel lenses," ACS Photonics (2019).

[6] Bertoncini, A. and Liberale, C., "3d printed high-na ultra-thin catadioptric condenser to minimize background contribution from cross-phase modulation in stimulated raman scattering microscopy (conference presentation)," in [Multiphoton Microscopy in the Biomedical Sciences XIX], 10882, 1088229, International Society for Optics and Photonics (2019).

[7] Dietrich, P., Göring, G., Trappen, M., Blaicher, M., Freude, W., Schimmel, T., Hölscher, H., and Koos, C., "3d-Printed Scanning-Probe Microscopes with Integrated Optical Actuation and Read-Out," Small, 1904695 (Dec. 2019).

[8] Hahn, V., Kalt, S., Sridharan, G. M., Wegener, M., and Bhattacharya, S., "Polarizing beam splitter integrated onto an optical fiber facet," Opt. Express 26, 33148-33157 (Dec 2018).

[9] Bertoncini, A. and Liberale, C., "Polarization micro-optics: circular polarization from a fresnel rhomb 3d printed on an optical fiber," IEEE Photonics Technology Letters 30(21), 1882-1885 (2018).

[10] Cojoc, G., Bertoncini, A., Müller, P., Guck, J., and Liberale, C., "Optical trapping and manipulation with a low na objective through 3d printed micro-mirrors (conference presentation)," in [Optical Trapping and Optical Micromanipulation XVI], 11083, 110830C, International Society for Optics and Photonics (2019).

[11] Guck, J., Ananthakrishnan, R., Mahmood, H., Moon, T. J., Cunningham, C. C., and Käs, J., "The optical stretcher: a novel laser tool to micromanipulate cells," Biophysical journal 81(2), 767-784 (2001).

[12] Guck, J., Schinkinger, S., Lincoln, B., Wottawah, F., Ebert, S., Romeyke, M., Lenz, D., Erickson, H. M., Ananthakrishnan, R., Mitchell, D., et al., "Optical deformability as an inherent cell marker for testing malignant transformation and metastatic competence," Biophysical journal 88(5), 3689-3698 (2005).

[13] Gansel, J. K., Thiel, M., Rill, M. S., Decker, M., Bade, K., Saile, V., von Freymann, G., Linden, S., and Wegener, M., "Gold helix photonic metamaterial as broadband circular polarizer," Science 325(5947), $1513-1515$ (2009).

[14] Waller, E. H. and von Freymann, G., "From photoinduced electron transfer to 3d metal microstructures via direct laser writing," Nanophotonics 7(7), 1259-1277 (2018). 